\title{
DIAGNOSTICS AND ANALYSIS OF INSTABILITIES WITH THE DIGITAL TRANSVERSE MULTIBUNCH FEEDBACK AT ELETTRA
}

\author{
L. Tosi, V. Smaluk, D. Bulfone, E. Karantzoulis, M. Lonza \\ Sincrotrone Trieste, 34012 Trieste, Italy
}

\begin{abstract}
The commissioning of the digital transverse feedback system at ELETTRA provides a valuable tool for the diagnosis and the study of transverse instabilities. The possibility of visualizing and analyzing the details of the time evolution enables the extraction of information regarding unstable and stable modes, such as growth and damping rates and their dependence on the harmonic sextupole. In this paper we present our first experience with the feedback system as a beam diagnostic tool both in multibunch and in single bunch operation modes.
\end{abstract}

\section{INTRODUCTION}

The digital multibunch transverse feedback [1] of ELETTRA consists in a wide-band bunch-by-bunch system where the positions of each of the 432 bunches can be acquired and eventually corrected. The wide band signals of the bunches, separated by $2 \mathrm{~ns}$, are demodulated into a base-band signal between 0 and $250 \mathrm{Mhz}$. The details of the electronics and its performance may be found in [2] and [3].

The signals acquired from the individual bunches are proportional to the position and to the current intensity of the bunches. This leads to the necessity of using software filters to eliminate both the DC component of the signals and the eventual influence on the signal of longitudinal oscillations. While the first is straightforwardly done by subtracting from each bunch signal its mean value over the number of turns which have been acquired, the latter may be eliminated with a software Butterworth filter. In this way, it is possible to clear the multibunch spectrum from the revolution harmonics and from eventual coherent longitudinal multibunch modes. In the near future, also the current intensity signal of the individual bunches will be available, enabling a more thorough and precise analysis.

The memory of the system is such that it allows the acquisition of the bunch positions in real time over a time span of $192 \mathrm{~ms}$ and thus over 200000 turns. The possibility of visualizing the details of beam motion over such a long time opens a whole new variety of measurements that were not previously available at ELETTRA. Particularly interesting is the possibility of analyzing the details of the time evolution of the dynamics of the beam in the presence of transverse coupled multibunch instabilities, which may permit a deeper understanding both of the nature of the modes as well as of the parameters of the machine which may influence them. Past observations have shown that the excitation level of the instabilities are related to the field strength of the harmonic sextupole [4]. In this paper, we present our first experiences in using the feedback system as a diagnostic tool, both for a deeper understanding of the effect of the harmonic sextupole as well as for beam measurements which previously could not be achieved. In section 2 multibunch measurements are presented while in section 3 with those done in single bunch.

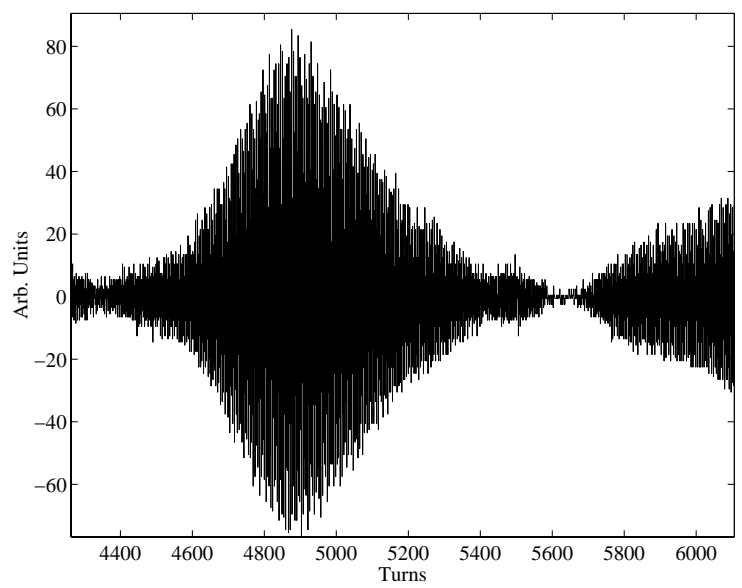

Figure 1. Horizontal beam motion of one bunch

\section{MULTIBUNCH MODE}

Multibunch analysis has mainly concentrated on further investigation of the effect of the harmonic sextupole in the horizontal plane. Figure 1 shows a typical motion of an individual bunch in the presence of horizontal multibunch instability with a spectrum shown in figure 2. The multibunch spectrum is obtained over 2000 turns in correspondence to the increase of the amplitude of the bunch oscillation. The main unstable modes are centered at $0.832 \mathrm{MHz}$ and $26.277 \mathrm{MHz}$ corresponding to the horizontal coupled mode numbers 417 and 394. Neither of

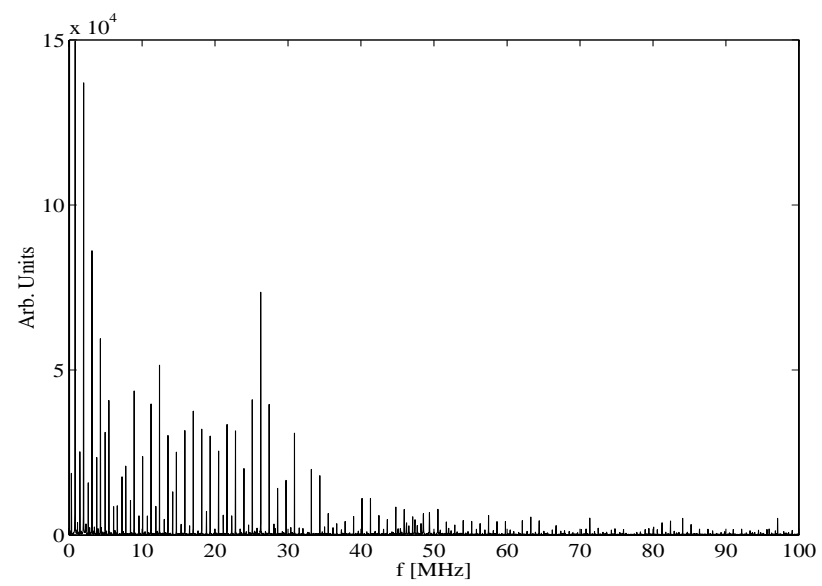

Figure 2. A typical horizontal multibunch spectrum 
the two modes correspond to known cavity higher order modes. One of the main problems in the analysis of the motion consists in the fact that the acquired signal can not be converted to the actual beam position because the system is still not calibrated.

Varying the harmonic sextupole changes the excitation level of the unstable modes with the maximum at $61 \mathrm{~A}$ and a clearance of the modes below $36 \mathrm{~A}$ and above $81 \mathrm{~A}$. Although past investigations [4] could be done only at a macroscopic level, a strong correlation was found between the horizontal instabilities and the coefficient $\mathrm{C}_{11}$ of the tune shift with amplitude induced by the harmonic sextupole:

$$
\begin{aligned}
& \Delta v_{\text {hor }}=C_{11} 2 J_{\text {hor }}+C_{12} 2 J_{\text {ver }} \\
& \Delta v_{\text {ver }}=C_{12} 2 J_{\text {hor }}+C_{22} 2 J_{\text {ver }}
\end{aligned}
$$

where $\mathrm{J}_{\text {hor/ver }}$ are the perturbed action variables. The results found had suggested the idea that this second order effect of the sextupole was generating a tune spread and thus giving a Landau damping of the oscillations.

The motion of individual bunches is very irregular and seemingly chaotic, due to the large number of modes involved, rendering its analysis as a function of the harmonic sextupole complicated. Thus the extraction of the rise times of an individual bunch motion is not sufficient to give an understanding of the mechanics involved, since these will depend heavily on the phases of the modes and on which one is most strongly influencing the motion at the moment. In this situation, an analysis of the evolution in time of the amplitudes of the unstable modes for various harmonic sextupole settings is more meaningful. It goes without saying that this was performed always during a time span in which horizontal beam motion was found to have increasing amplitudes. The rise and damping times of the unstable modes as a function of the harmonic sextupole settings are shown in figure 3. It can be observed that there is a concentration of unstable modes with positive rise times at those values for which the absolute value of $\mathrm{C}_{11}$ is near its theoretical minimum (see figure 4). Furthermore, as the two sextupole settings where the beam is stable are approached, a larger number of unstable modes decrease in

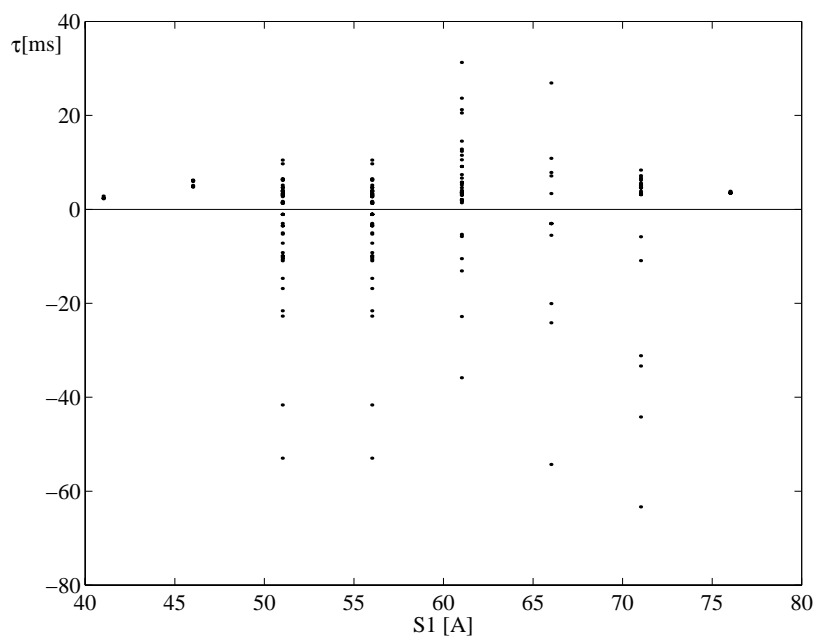

Figure 3. Rise and damping times of the unstable modes as a function of the harmonic sextupole settings

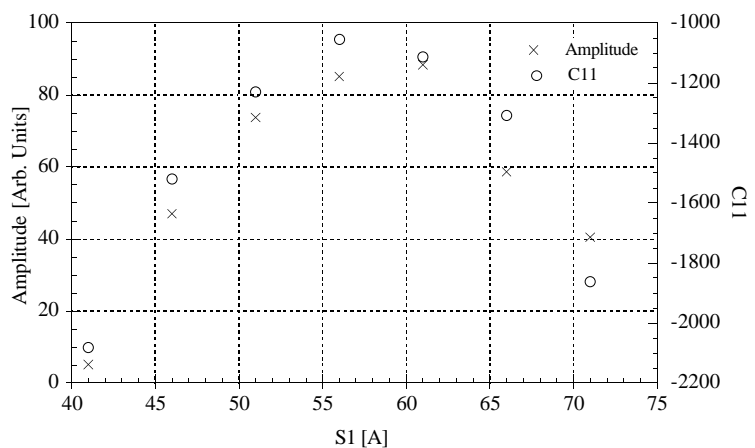

Figure 4. Comparison of the maximum horizontal amplitude of the bunch's motion with $\mathrm{C}_{11}$ as functions of the harmonic sextupole settings

amplitude and are damped. At the same time, the maximum measured amplitudes of the individual bunch vary as shown in figure 4 , where also the theoretical behaviour of the coefficient $\mathrm{C}_{11}$ is illustrated, confirming the results of [4].

The tune spread required for Landau damping may be computed with $\Delta v=\left(\pi f_{o} \tau\right)^{-1}$, where $f_{o}$ is the revolution frequency and $\tau$ the rise time. It results that for the fastest mode with $\tau=3.5 \mathrm{~ms}$ the tune spread required is $7.8 \cdot 10^{-5}$. The Landau damping would require the condition that $2 J_{\text {hor }}=\left(C_{11} \pi f_{o} \tau\right)^{-1}$, corresponding to an oscillation amplitude of $3 \sigma_{\text {hor }}$. Qualitative observations of the image of the unstable beam on the profile monitor suggested that the amplitudes were of this order of magnitude. We intend to confirm the mechanism in a situation in which the instability is driven by only one high order dipole mode of a cavity.

\section{SINGLE BUNCH MODE}

Data measured with the system have been used to estimate the transverse coupling impedance. The measurements were performed in single bunch mode at the injection energy of $E=0.9 \mathrm{GeV}$, with betatron tunes of $v_{\text {hor }}=14.29, \quad v_{v e r}=8.19$, and with chromaticities of $\xi_{\text {hor }} \cong \xi_{\text {ver }} \cong 0.5$.

The impedance has both a resistive $\operatorname{Re} Z_{\perp}$ and a reactive $\operatorname{Im} Z_{\perp}$ component. The resistive part causes a fast damping of the betatron oscillations, which is proportional to the beam current $I$ as [5]:

$$
\frac{1}{\tau}=\frac{I c^{2} \cdot \operatorname{Im} f(2 \chi)}{32 \pi^{2}(E / e) v f_{0} b} \cdot \operatorname{Re} Z_{\perp}
$$

where $E / e$ is the particle energy in electron-volts, $v$ the betatron tune, $f_{0}$ the revolution frequency, $b$ the average vacuum chamber radius, and $c$ the speed of light. $f(2 \chi)$ is the the complex function $f(u)=\int_{0}^{\pi} e^{i u \sin x} d x$ of the headtail phase $\chi=\frac{2 \pi v f_{0} \xi \sigma_{s}}{c \alpha}$, where $\xi=\frac{\Delta v}{\Delta p / p}$ is the chromaticity, $\sigma_{s}$ the r.m.s. of the bunch length, and $\alpha$ the momentum compaction. 


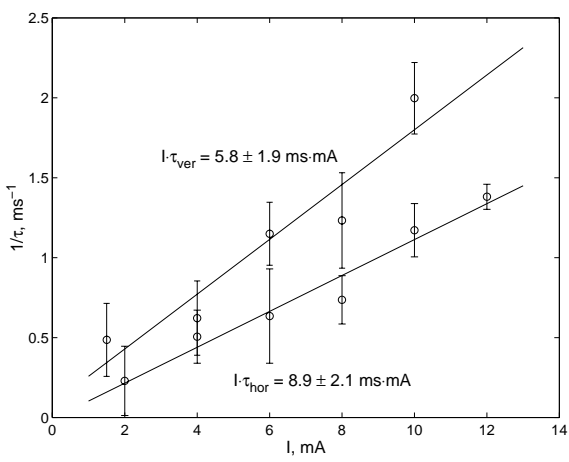

Figure 5: Fast damping rates

For various beam currents, the damping rates were estimated by analyzing the turn-by-turn beam positions measured with the system. Whereas coherent horizontal betatron oscillations were excited by the injection kickers, resonance excitation was used to produce vertical oscillations. In a near future beam excitation will be done by the system operating in the anti-damping mode. The damping rates vs. beam current are shown in figure 5. On the basis of these measurements, the resistive part of the transverse impedance results to be $\operatorname{Re} Z_{\perp}=0.16 \pm 0.05 \mathrm{M} \Omega / \mathrm{m}$.

The reactive part $\operatorname{Im} Z_{\perp}$ of the transverse impedance produces a current dependent coherent shift of the betatron tunes [5]:

$$
\frac{\Delta v}{\Delta I}=-\frac{R}{4 \pi \sigma_{s}(E / e)} \cdot \sum_{i=1}^{N} \beta_{i} \operatorname{Im} Z_{\perp i}
$$

where $\beta_{i}$ is the beta function at the $i$-th impedance location, $R$ the ring radius and $\sigma_{s}$ the r.m.s. of the bunch length. Both the horizontal and the vertical coherent tune shifts were measured using the above-mentioned measurement technique (see figure 6). The inter-harmonic method [6] of FFT refinement was implemented for the data processing, which improved the accuracy of the tune measurement to the value of $<2.0 \cdot 10^{-4}$. The vertical tune shift $\Delta v_{v e r} / \Delta I=-0.78 \pm 0.02 A^{-1}$ is much greater than the horizontal one $\Delta v_{\text {hor }} / \Delta I=-0.16 \pm 0.02 A^{-1}$, due to the reduced physical aperture in the vertical plane. The total reactive transverse impedance, calculated from these precise tune shift measurements and taking the measured $\sigma_{s}=7.5 \mathrm{~mm}, \quad$ is $\operatorname{Im} Z_{\perp}=0.4 \pm 0.02 \mathrm{M} \Omega / \mathrm{m}$. This value is in good agreement with the previously measured one [7].
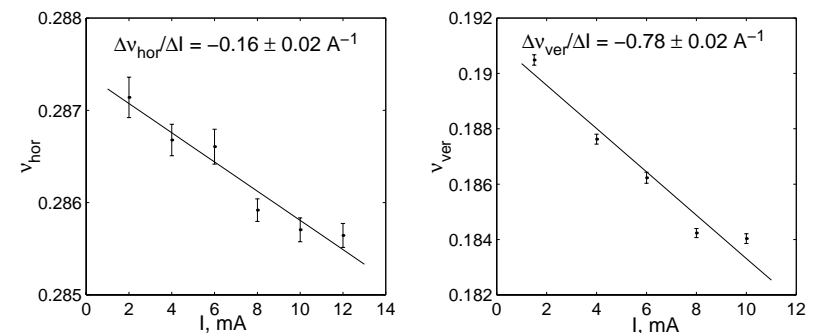

Figure 6: Coherent tune shift.

\section{CONCLUSIONS}

First experience in using the digital transverse feedback system as a diagnostic tool has been very positive. Although the system still is lacking the beam intensity signal and knowledge of the absolute value of the data (features which will available in the near future), it is retained as a very precious tool for investigating beam dynamics in the storage ring. Previous results on the effects of the harmonic sextupole on the excitation level of horizontal instabilities have been confirmed. In single bunch mode more precise measurements of the tune shift with current as well as the measurement of the fast coherent damping time due to the real part of the impedance were made possible. As the performance of the system as a diagnostic tool improves, in the future we intend to continue investigation with the harmonic sextupole both in multibunch and in single bunch modes measuring its effect on the Landau damping tune spread and on the fast coherent damping. The system will also reveal itself to be a precious tool in investigating the unknown nature and source of the unstable modes.

\section{REFERENCES}

[1] D. Bulfone et al., "design Considerations for the ELETTRA Transverse Multi-Bunch Feedback", PAC-99, New York, March 1999.

[2] M. Lonza et al., "Digital Processing Electronics for the ELETTRA Transverse Multi-Bunch Feedback System", ICALEPCS-99, Trieste, October 1999.

[3] D. Bulfone et al., "Exploitation of the Integrated Digital Processing and Analysis of the ELETTRA/SLS Transverse Multibunch Feedback System", this conference.

[4] L. Tosi and E. Karantzoulis, "Effects of the Harmonic Sextupole on Transverse Multibunch Instabilities at ELETTRA", PAC-2000, New York, March 2000

[5] A.Chao, "Physics of collective beam instabilities in high energy accelerators", John Wiley \& Sons, Inc. 1993

[6] A. Kalinin, V. Smaluk, "Data processing for turn-byturn beam position monitor", HEACC-98, Dubna, September 1998.

[7] E. Karantzoulis, "The evolution of the impedance of Elettra and related instabilities", Sincrotrone Trieste Technical Note, ST/M-TN-00/8. 